\title{
Low-complexity DBP using optical-field-intensity averaging for digital coherent system
}

\author{
Shin Takano ${ }^{1,}$ a) and Hiroyuki Uenohara ${ }^{1}$ \\ ${ }^{1}$ Laboratory for Future Interdisciplinary Research of Science and Technology \\ (FIRST), Tokyo Institute of Technology, 4259 Nagatsuta-cho, Midori-ku, Yokohama, \\ Kanagawa 226-8503, Japan
}

a)takano.s.ag@m.titech.ac.jp

\begin{abstract}
We numerically assess the performance and computational complexity of split-step Fourier method-based digital backpropagation with optical-field-intensity averaging (FIA-DBP) for reducing digital signal processing complexity in an optical receiver. We propose FIA-DBP to effectively compensate for nonlinear distortions due to symbol interactions. FIA-DBP averages the optical field intensity estimated in every span from received signals. We show the numerical results for 56 Gbaud single carrier dual polarization64 quadrature amplitude modulation signals. Our proposed method achieves a complexity $50.8 \%$ less than that of conventional digital backpropagation at a transmission distance of $2400 \mathrm{~km}$ and a launched power of $-2 \mathrm{dBm}$.
\end{abstract}

Keywords: digital backpropagation, digital signal processing, coherent optical communication, nonlinear effect, low complexity

Classification: Fiber-Optic Transmission for Communications

\section{References}

[1] M. Secondini and E. Forestieri, "Scope and limitations of the nonlinear Shannon limit,” IEEE J. Lightw. Technol., vol. 35, no. 4, pp. 893-902, Feb. 2017. DOI: 10.1109/JLT.2016.2620721

[2] E. Ip and J.M. Kahn, "Compensation of dispersion and nonlinear impairments using digital backpropagation,” IEEE J. Lightw. Technol., vol. 26, no. 20, pp. 3416-3425, Oct. 2008. DOI: 10.1109/JLT.2008.927791

[3] D.S. Millar, S. Makovejs, C. Behrens, S. Hellerbrand, R.I. Killey, P. Bayvel, and S.J. Savory, "Mitigation of fiber nonlinearity using a digital coherent receiver," IEEE J. Sel. Top. Quantum Electron., vol. 16, no. 5, pp. 1217-1226, Sep. 2010. DOI: 10.1109/JSTQE.2010.2047247

[4] T. Hoshida, L. Dou, T. Tanimura, W. Yan, S. Oda, L. Li, H. Nakashima, M. Yan, Z. Tao, and J.C. Rasmussen, "Digital nonlinear compensation techniques for high-speed DWDM transmission systems," Proc. SPIE, San Francisco, California, vol. 8284, 82840 P-10, Jan. 2012. DOI: 10.1117/12.907842

[5] M. Secondini, D. Marsella, and E. Forestieri, "Enhanced split-step Fourier method for digital backpropagation," 2014 The European Conference on Optical Communication (ECOC), We.3.3.5, Sep. 2014. DOI: 10.1109/ECOC.2014. 6964122

[6] S.B. Amado, F.P. Guiomar, N.J. Muga, R.M. Ferreira, J.D. Reis, S.M. Rossi, A. Chiuchiarelli, J.R.F. Oliveira, A.L. Teixeira, and A.N. Pinto, "Low complexity 
advanced DBP algorithms for ultra-long-haul $400 \mathrm{G}$ transmission systems," IEEE J. Lightw. Technol., vol. 34, no. 8, pp. 1793-1799, April 2016. DOI: 10.1109/JLT.2015.2512038

[7] H. Yao, W. Li, J. Han, Z. He, Z. Yuan, Q. Hu, Q. Yang, and S. Yu, "A modified adaptive DBP for DP 16-QAM coherent optical system," IEEE Photon. Technol. Lett., vol. 28, no. 22, pp. 2511-2514, Nov. 2016. DOI: 10.1109/ LPT.2016.2601933

[8] C. Fougstedt, L. Svensson, M. Mazur, M. Karlsson, and P. Larsson-Edefors, "ASIC implementation of time-domain digital back propagation for coherent receivers," IEEE Photon. Technol. Lett., vol. 30, no. 13, pp. 1179-1182, July 2018. DOI: 10.1109/LPT.2018.2837349

[9] H. Rabbani, H. Rabbani, L. Beygi, and E. Agrell, "Improving the achievable rates of optical coherent transmission with back-propagation," IEEE Photon. Technol. Lett., vol. 30, no. 14, pp. 1273-1276, July 2018. DOI: 10.1109/LPT. 2018.2839652

[10] S. Takano and H. Uenohara, "Low computational complexity of optical nonlinear compensation with phase linear approximation method for single polarization-64QAM signals," OECC/PSC 2019, TuP4-B3, July 2019. DOI: 10.23919/PS.2019.8817813

[11] S. Takano and H. Uenohara, "Low complexity DBP with phase linear approximation for DP-16QAM coherent optical communication systems," IEICE Commun. Express, vol. 8, no. 8, pp. 329-334, Aug. 2019. DOI: 10.1587/comex. 2019XBL0072

[12] F. Yaman and G. Li, "Nonlinear impairment compensation for polarizationdivision multiplexed WDM transmission using digital backward propagation," IEEE Photon. J., vol. 1, no. 2, pp. 144-152, Aug. 2009. DOI: 10.1109/JPHOT. 2010.2071860

[13] R. Schmogrow, B. Nebendahl, M. Winter, A. Josten, D. Hillerkuss, S. Koenig, J. Meyer, M. Dreschmann, M. Huebner, C. Koos, J. Becker, W. Freude, and J. Leuthold, "Error vector magnitude as a performance measure for advanced modulation formats," IEEE Photon. J., vol. 24, no. 1, pp. 61-63, Jan. 2012. DOI: 10.1109/LPT.2011.2172405

\section{Introduction}

Spectral efficiency is severely limited by the impact of nonlinear distortion due to the optical Kerr effect in optical communication systems [1]. Digital backpropagation (DBP) is one of the most effective methods for compensating nonlinear distortions, and advanced split-step Fourier method (SSFM)-based DBP algorithms have been investigated thus far $[2,3,4,5]$. However, owing to the highly iterative algorithm involved, DBP has been regarded as computationally complex for real-time processing, which is one of the significant technical issues in optical communication systems. Hence, several low-complexity and advanced DBP algorithms have been proposed and the complexity has been reduced $[6,7,8,9]$. Despite these significant efforts, we believe the DBP algorithm can be further improved for implementation in low-power digital signal processors. Therefore, we have proposed phase linear approximation-DBP (PLA-DBP) as a candidate [10, 11]. PLA-DBP estimates the phase of input signals by performing a linear approximation in each span. However, in PLA-DBP, the transmission distance is limited because the effect of nonlinear 
intensity distortion is not sufficiently compensated. Therefore, we further propose optical-field-intensity-averaging DBP (FIA-DBP), which averages the optical field intensity estimated at every span from received signals. FIA-DBP enables the symbol at the transmission to be estimated efficiently because nonlinear distortions can be mitigated by averaging.

We herein numerically compare the performances and complexities of FIA-DBP for 28 Gbaud and 56 Gbaud single-carrier dual polarization-64 quadrature amplitude modulation (DP-64QAM) transmission systems with the conventional SSFM-based DBP.

\section{Principle of optical-field-intensity averaging and simulation setup}

DBP is based on the concept that original signals are estimated from received signals propagating in the direction opposite the transmission direction through a virtual fiber. In polarization division multiplexed optical transmission systems, the evolution of optical field envelopes is modeled by the Manakov equation shown in Eq. (1). It is derived by averaging the vectoral nonlinear Schrödinger equation over the Poincaré sphere, considering that random birefringence fluctuations in optical fibers occur much faster than nonlinear interactions [12].

$$
\frac{\partial E_{x / y}}{\partial z}=-\frac{\alpha}{2} E_{x / y}-i \frac{\beta_{2}}{2} \frac{\partial^{2} E_{x / y}}{\partial t^{2}}+i \frac{8}{9} \gamma\left(\left|E_{x}\right|^{2}+\left|E_{y}\right|^{2}\right) E_{x / y},
$$

where $E_{x / y}$ represents the optical field envelope for two orthogonal $x$ and $y$ polarizations; $\alpha, \beta_{2}$, and $\gamma$ are the attenuation coefficient, group velocity dispersion, and nonlinear coefficients of the fiber, respectively; $z$ and $t$ denote the propagation direction and time, respectively.

Figure 1 (a) shows the block diagram of FIA-DBP. In each step, $E_{x / y}$ is nonlinearly compensated by DBP over the entire span. In each span, $E_{x / y}$ is stored in the parameter $E_{x / y, D B P, N_{s p a n}}$, and the field intensity is stored in the memory after the residual linear distortions between the span and launched point are compensated. After performing these processes in all steps and spans, the final optical field intensity $E_{x / y, F I A-D B P}$ is estimated by averaging $E_{x / y, D B P, N_{s p a n}}$ using Eq. (2),

$$
E_{x / y, F I A-D B P}=\left(\sum_{N_{\text {span }}} E_{x / y, D B P, N_{\text {span }}}\right) / N_{\text {span }} .
$$

where $N_{\text {span }}$ denotes the number of spans.

Figure 1 (b) illustrates the simulation setup used in this study. In the transmitter, $2^{21}$ - 1 pseudo-random binary sequence (PRBS) codes are generated, and DP64QAM signals are transmitted at a carrier wavelength of $1550 \mathrm{~nm}$. The modulation speed is 28 or 56 Gbaud. The signals are transmitted through standard singlemode fiber (SSMF), and the loss is compensated using erbium doped fiber amplifier (EDFAs) installed at a $100 \mathrm{~km}$ span with an optical gain of $16 \mathrm{~dB}$ and noise figure of $5 \mathrm{~dB}$. The parameters of the SSMF are as follows: loss of $0.16 \mathrm{~dB} / \mathrm{km}$; dispersion parameter of $16 \mathrm{ps} / \mathrm{nm} / \mathrm{km}$; nonlinear coefficient of $1.3 / \mathrm{W} / \mathrm{km}$; and core radius of $5.0 \mu \mathrm{m}$. In the receiver, after a coherent reception using a local oscillator with a laser linewidth of $100 \mathrm{kHz}$, linear and nonlinear compensation is performed, and the $Q$ 


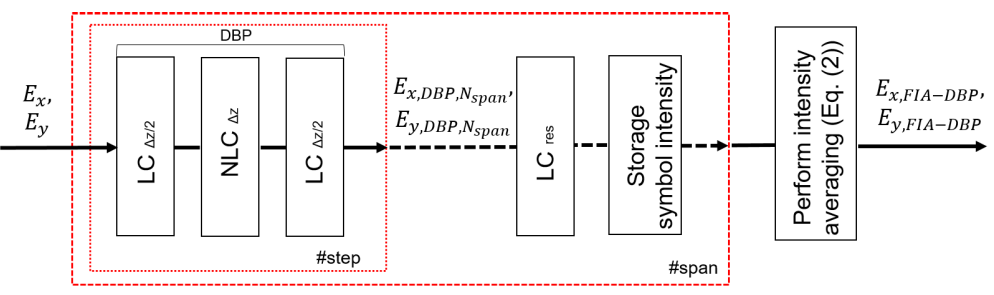

(a)

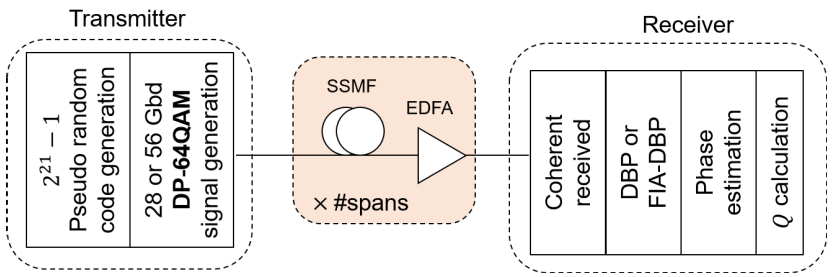

(b)

Fig. 1. System model. (a) Block diagram of FIA-DBP. LC: linear compensation; NLC: nonlinear compensation; LCres: residual linear compensation. (b) Simulation setup. Number of transmission steps: 100; number of data samples/symbol: 16 .

value is calculated after phase estimation, defined as $Q=20 \cdot \log _{10}(\sqrt{2} \cdot \operatorname{erfcinv}(2$. $B E R)$ ), where erfcinv is an inverse complementary error function. The bit error ratio (BER) is calculated by using Eqs. (3) and (4).

$$
B E R \sim \frac{\left(1-L^{-1}\right)}{\log _{2} L} \operatorname{erfc}\left[\sqrt{\frac{3 \log _{2} L}{\left(L^{2}-1\right)} \frac{\sqrt{2}}{(k E V M)^{2} \log _{2} M}}\right],
$$

where

$$
k=\frac{\left|S_{t, \max }\right|}{\sum_{i=1}^{M}\left(\left|S_{t, i}\right| / M\right)} .
$$

Here, $L$ is the number of signal levels within each orthogonal direction of the constellation diagram, $\log _{2} M$ is the number of bits encoded into one QAM symbol, $S_{t, i}$ is the ideal transmitted field vector, and $S_{t, \max }$ is the field vector of the outermost constellation point [13].

Figure 2 shows the fundamental properties of the proposed FIA-DBP. Figure 2 (a) indicates the field intensity transition per span of 64QAM signals with field intensity from the fourth until the maximum in a case involving compensation by DBP. As shown, the field intensity fluctuated, and the fluctuation was not sufficiently small as to correctly estimate the input signal owing to the nonlinear distortion caused, as expressed in Eq. (1), ASE noise generated in EDFAs and their interactions, and the interference between adjacent signals. Owing to such fluctuations, the target symbol will be misjudged as the incorrect adjacent symbol on the constellation map at the receiver.

The results of introducing FIA-DBP to solve the fluctuation and comparing it with that of DBP is shown in Fig. 2 (b). In the case of FIA-DBP, nonlinear compensation for each step was applied to the optical field intensity, and the average value $E_{x / y, F I A-D B P}$ of $E_{x / y, D B P, N_{\text {span }}}$ obtained using Eq. (2) at each span are plotted. Here, the field intensity of all ideal 64QAM symbols was normalized to 1.00. At the last span number 12, the normalized field intensity of the symbol 


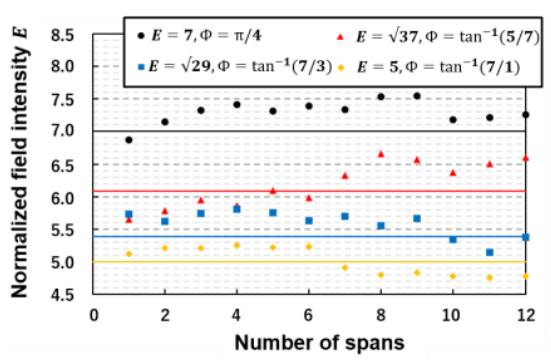

(a)

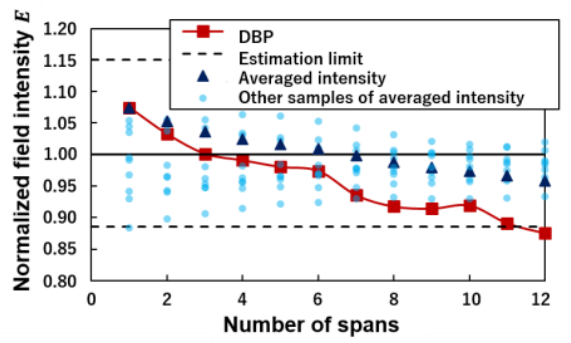

(b)

Fig. 2. Fundamental properties of FIA-DBP; baud rate of 28 Gbaud, transmission distance of $1200 \mathrm{~km}$, launched power of $-3 \mathrm{dBm}$; (a) intensity transition per span by DBP; the smallest intensity of 64QAM is defined as $\mathrm{E}=1.00$, showing samples from the fourth until the maximum. (b) Concept of intensity averaging; all optical field intensity of 64QAM symbols are normalized to 1.00. Estimation limit is defined as correct/incorrect decision boundary between the target field intensity and the incorrect adjacent field intensity defined on the constellation map.

using DBP ( $\square$ ) exceeded the estimation limit of the adjacent field intensity (dotted line), and an error occurred. When the field intensity was averaged successively for each span while performing FIA-DBP, significant changes in field intensity were suppressed as shown by $(\boldsymbol{\Delta})$; therefore, the field intensity can be estimated correctly. In addition, the field intensity of the other 10 samples $(\bigcirc)$ reached the reference value. This indicates that nonlinear distortions can be effectively reduced using the optical field intensity averaging method.

\section{Numerical results}

The performance and complexity after compensation by FIA-DBP are shown in Fig. 3. PRBS codes of length $2^{21}-1$ was used for DP-64QAM.

Figure 3 (a) shows the $Q$ value vs. launched power when linear compensation (LC), DBP, and FIA-DBP were performed at the transmission distance of $1200 \mathrm{~km}$. The insets show the constellations for signals at $56 \mathrm{Gbaud}, \mathrm{LC}, N_{\text {step,DBP }}=15$ and $N_{\text {span,DBP }}=12$ for DBP, $N_{\text {step }}=15$, and $N_{\text {span }}=12$ for FIA-DBP. The $Q$ value decreased with increasing baud rate from 28 to $56 \mathrm{Gbaud}$, and the peak power yielding the maximum $Q$ value increased from -3 to $-1 \mathrm{dBm}$. Furthermore, the peak power improved by $2 \mathrm{~dB}$ from LC using FIA-DBP. At $56 \mathrm{Gbaud}$, the $Q$ values of FIA-DBP were 1.17 and $0.15 \mathrm{~dB}$ better than those of LC and DBP, respectively. Provided that the $Q$ value of IA-DBP is similar to that of DBP, a comparable constellation map can be obtained.

In Fig. 3 (b), $Q$ value vs. $N_{\text {span }}$ is shown under the conditions of a transmission distance of $1200 \mathrm{~km}$, as well as launched powers of -3 (28 Gbaud) and $-1 \mathrm{dBm}$ (56 Gbaud). As shown, the $Q$ value of IA-DBP improved compared with that of DBP when $N_{\text {span }}$ increased. Under such conditions, it was confirmed that the $Q$ value of FIA-DBP improved compared with that of DBP. In addition, a small $N_{\text {span }}$ (approximately $N_{\text {span }}=10$ ) was sufficient to maintain the same $Q$ value as that of DBP for both baud rates.

The reduction rate of computational complexity using FIA-DBP compared with 


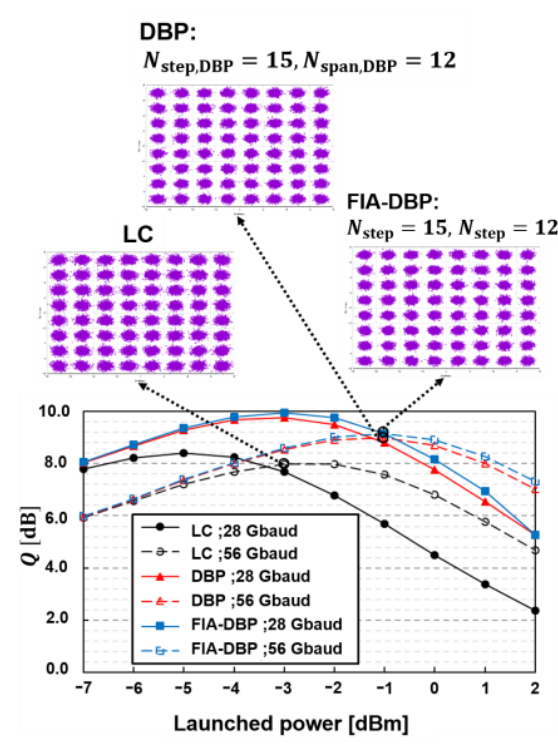

(a)

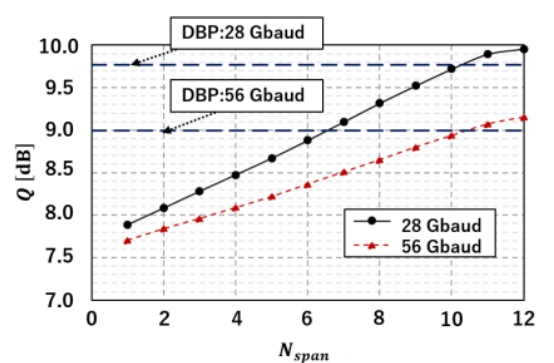

(b)

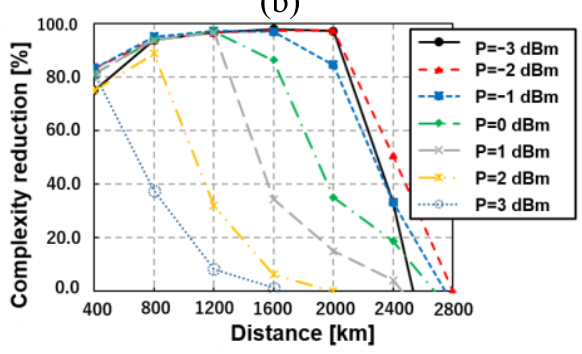

(c)

Fig. 3. Numerical results of FIA-DBP; (a) $Q$ value vs. launched power; transmission distance of $1200 \mathrm{~km}$; insets: constellations of symbols at $56 \mathrm{Gbaud}$; LC, DBP with $N_{\text {step,DBP }}=15$ and $N_{\text {span,DBP }}=12$, and FIA-DBP with $N_{\text {step }}=15$ and $N_{\text {span }}=12$. (b) $Q$ value vs. $N_{\text {span }}$ of FIA-DBP; transmission distance of $1200 \mathrm{~km}$, launched power is set at the peak power of Fig. 3 (a). (c) Reduction rate of computational complexity by FIA-DBP compared with DBP; target BER of $2.4 \times 10^{-2}(Q=5.7 \mathrm{~dB})$.

that using DBP is summarized in Fig. 3 (c). The reduction rate reaching the target BER of $2.4 \times 10^{-2}(Q=5.7 \mathrm{~dB})$ is calculated using the following equation:

$$
\text { Reduction rate }=1-\frac{N_{\text {step }} \times N_{\text {span }}}{N_{\text {step,DBP }} \times N_{\text {span,DBP }}} .
$$

where $N_{\text {step,DBP }}$ and $N_{\text {span,DBP }}=($ Transmission distance $L) /($ span length $)$ are the number of steps and spans required for DBP to achieve the target BER, respectively. The transmission distance $L$ varied from 400 to $2800 \mathrm{~km}$, and the launched power $P$ varied from -3 to $3 \mathrm{dBm}$. The maximum reduction rate was obtained from the optimized combination of $N_{\text {step }}$ and $N_{\text {span }}$. When the nonlinearity was relatively small, FIA-DBP exerted a significant effect; for example, FIA-DBP enabled the computational complexity to be reduced by $50.8 \%$ with the same performance as that of DBP when $L=2400 \mathrm{~km}$ and $P=-2 \mathrm{dBm}$. Even at a high power, computational complexity reduction can be achieved through FIA-DBP; for example, a reduction of $37.5 \%$ when $L=800 \mathrm{~km}$ and $P=3 \mathrm{dBm}$.

\section{Conclusion}

We reported the performances and complexities of FIA-DBP for 56 Gbaud DP64QAM systems based on simulation. At 56 Gbaud, the $Q$ values of FIA-DBP were 1.17 and $0.15 \mathrm{~dB}$ better than those of LC and DBP, respectively. The computational complexity and $Q$ value of FIA-DBP were tradeoffs between $N_{\text {step }}$ and $N_{\text {span }}$, whereas a reduction of complexity can be expected under the optimal combination. Under the conditions of $L=2400 \mathrm{~km}$ and $P=-2 \mathrm{dBm}$, the computational complexity can be reduced by $50.8 \%$ while maintaining the $Q$ value. 